\title{
Development of a compact scintillator hodoscope with wavelength-shifting fibre read-out
}

\author{
C. P. Achenbach* and J. H. Cobb \\ University of Oxford, Sub-department of Particle Physics, Denys Wilkinson Bld., \\ Keble Rd., Oxford, OX1 3RH, UK
}

\begin{abstract}
We report on the prototyping of a plastic scintillator hodoscope with wavelengthshifting fibre read-out by a multi-anode photomultiplier as part of the development of a detector for cosmic ray muons to be carried aboard an aircraft. Light yield and light attenuation measurements on single- and double-clad wavelength-shifting fibres were performed. Low power, low-threshold, discriminators were designed. A prototype 16-channel hodoscope with two planes was built and tested with cosmic rays. After correcting for geometrical factors a global intrinsic efficiency of $\epsilon>98 \%$ was obtained in both planes. The overall performance of the hodoscope proved it to be well suited for the ADLER experiment to measure the high altitude muon flux.
\end{abstract}

Key words: Cosmic ray detectors, Hodoscopes, Scintillation detectors, Wavelength-shifting fibres, Multi-anode photomultipliers

PACS: 29.40.Mc, 95.55.Vj, 85.60.Ha

\section{Introduction}

We report on the development of a plastic scintillator hodoscope with wavelengthshifting (WLS) fibre read-out by a multi-anode photomultiplier (PMT). The work described in this paper was part of the research and development activity for the ADLER experiment (iㅏborne Detector for Low Energy Rays) [1]. The

\footnotetext{
* Corresponding author. Present address: Institut für Kernphysik, Joh. GutenbergUniversität Mainz, J J Becher-Weg 45, 55099 Mainz, Germany. Tel.: +49-61313925831; fax: +49-6131-3922964.

Email address: patrick@kph.uni-mainz.de (C. P. Achenbach).
}

Preprint submitted to Nucl. Instr. and Meth. in Phys. Res. A 15 October 2018 
object of the ADLER experiment is to measure the flux of low energy atmospheric muons at aircraft cruising altitudes as a function of geomagnetic coordinates. Observations of muons at ground level are of limited value because of the large and unknown amount of kinetic energy (typically $2 \mathrm{GeV}$ ) lost by the muons in the atmosphere. While ground measurements are widely reported in the literature, there have been very few attempts to measure the muon flux as a function of altitude and latitude. The effect of the geomagnetic field is recognised as very important in cosmic ray cascade calculations. Accordingly, the proposed measurement could provide an important calibration of the calculations of atmospheric neutrino fluxes performed by the Bartol group [2],[3], and by Honda, Kajita, Kasahara and Midorikawa [4]. Accurate predictions of the neutrino fluxes are needed in the analysis of data from underground experiments such as Super-Kamiokande [5], MACRO [6] and Soudan 2 [7] to obtain a better understanding of flavour oscillations of muon neutrinos.

Section 2 describes the design criteria for the hodoscope which follow from the special measurement conditions of the proposed ADLER experiment. Simple estimates which guided the design of the hodoscopes and front-end electronics are given in Section 3. The light yield and light attenuation measurements on single- and double-clad WLS fibres which were performed to optimise the design of the hodoscopes and electronics are described in Section 4. A prototype 16-channel hodoscope with associated discriminators boards was constructed and tested to verify the performance of the design. In Section 5 we present the results of cosmic ray tests of this hodoscope.

\section{Design Considerations}

The aim of the initial phase of the ADLER experiment was to design a compact detector which could be carried by an aircraft to measure the muon flux at altitudes close to the production maximum at different geomagnetic locations. Severe constraints on mass, size, power consumption and efficiency of the instrument are imposed by the requirement that it could be carried by a commercial aircraft. The whole apparatus must be certified as meeting rigorous airworthiness requirements (specified in "Eurocae ED-14D"). For example, it must be designed to withstand forward accelerations of up to $9 \mathrm{~g}$ and lateral accelerations of $3 \mathrm{~g}$.

The conceptual ADLER detector consists of scintillator hodoscopes interleaved with a passive lead absorber followed by an active scintillator absorber surrounded by a veto counter. The individual counters must be highly efficient for an absolute measurement of the atmospheric muon count rate. Furthermore, the detector as a whole must provide sufficient spatial resolution to correlate hits in a number of hodoscope planes and to assign them to unique 
muon tracks. The inner components of the detector are shown in Fig. 1. The active area of each hodoscope was chosen to be $A \approx 800 \mathrm{~cm}^{2}$. Muons which stop in the absorber are distinguished by the observation of delayed coincidences between signals from absorber and the hodoscopes and no activity in the surrounding veto-counters.

The ADLER detector requires of the order of 100 individual hodoscope channels. The use of conventional scintillation counters and single channel PMTs would be costly in terms of space and weight. It was therefore decided to base the design on the use of scintillators with WLS readout in order to achieve a compact detector. The WLS fibres serve the dual purpose of primary light collection and subsequent flexible light transmission over a distance of 50 $100 \mathrm{~cm}$. The drawback of the use of WLS fibres for primary light collection is that the overall 'light yield' (photo-electrons per unit energy deposit) is low compared with conventionally instrumented scintillation counters and it was necessary to undertake an optimisation study to ensure that efficient hodoscopes could be built.

The electronic read-out of the hodoscopes also had to be economical in terms of cost, space and power consumption. A main consideration was the avoidance of high power consuming electronic crates and modules. In addition, the use of multi-anode photomultiplier tubes greatly reduces the number of high-voltage (HV) power supplies and voltage dividers required. A compact tube like the Hamamatsu Photonics [8] R5900-00-M16 multi-anode photomultiplier (M16) fitted very well into the over-all scheme. The performance of the M16 tube was first reported in [9] and comprehensive tests on gain, uniformity, cross-talk and behaviour in magnetic fields have been conducted by [10]. Since this study, Hamamatsu has continued to make improvements to the tube particularly in quantum efficiency and collection efficiency of the dynode chain. The M16 has a $0.8 \mathrm{~mm}$ thick borosilicate window spaced only $2 \mathrm{~mm}$ from the first dynode, a single $17.5 \times 17.5 \mathrm{~mm}^{2}$ bialkali or S10 photocathode, individual metal channel dynodes and the output signals are obtained from independent anodes. The tube can be operated at negative $\mathrm{HV}$ s for dc-coupled anode read-out. It has a typical gain of about $1-3 \times 10^{6}$ when operated at $-800 \mathrm{~V}$. The $4 \times 4 \mathrm{~mm}^{2}$ pixels are arranged in a $4 \times 4$ matrix and separated by gaps of $0.275 \mathrm{~mm}$. The gain variation between pixels can be up to a factor of three. Despite this potential difficulty, its compact geometry of only $22 \times 30 \times 30 \mathrm{~mm}^{3}$ made it very attractive for our application. Six M16 PMTs are sufficient to perform the experiment. 


\section{Design Estimates}

\subsection{Light Yield}

A minimum ionising particle typically loses $\sim 1.8 \mathrm{MeV}$ of energy per $\mathrm{cm}$. of plastic scintillator. The energy deposited is converted to (blue) scintillation photons with a typical efficiency of $\epsilon_{s} \sim 1.5 \times 10^{4}$ photons $/ \mathrm{MeV}$. These photons will be absorbed efficiently and re-radiated at a longer wavelength (green) if they strike a WLS fibre. Since the size of a fibre is small compared with the dimensions of a scintillator bar, the probability that a blue photon will hit the fibre in one traversal of a bar is small, $P_{W} \sim d / s$, where $d$ is the diameter of the fibre and $s$ is the projected width of the scintillator bar. Blue photons may also be absorbed with a probability $P_{R}=1-R$ when they strike the wall of the scintillator bar, where $R$ is the reflection coefficient, or by bulk absorption with a probability $P_{A}$ in the scintillator. Therefore the

probability that the ultimate fate of a blue photon is to be absorbed by the WLS fibre is $\epsilon_{W}=P_{W} /\left(P_{W}+P_{R}+P_{A}\right)$. Choosing $d=1.2 \mathrm{~mm}, s=55 \mathrm{~mm}$ (corresponding roughly to a bar with a $20 \mathrm{~mm} \times 35 \mathrm{~mm}$ cross-section) and assuming $1-R=0.04$ and $P_{A}=0.02, \epsilon_{W}$ evaluates to $\sim 0.24$.

The green photons are re-radiated isotropically in the WLS fibre and only a small fraction, $\epsilon_{t}$ (typically 0.03 in a single clad fibre), are captured within the acceptance cone of the fibre and transmitted by internal reflection. The quantum efficiency, $\epsilon_{Q}$, of a bialkali photocathode is $\sim 0.1$ for green photons. Assuming no further loss of light, e.g. due to absorption in the fibre or losses at optical couplings, the light yield of a system using $20 \mathrm{~mm} \times 20 \mathrm{~mm}$ scintillator bars and $1.2 \mathrm{~mm}$ diameter fibres is expected to be $Y_{l}=\epsilon_{s} \epsilon_{W} \epsilon_{t} \epsilon_{Q} \approx$ $1.5 \times 10^{4} \times 0.24 \times 0.03 \times 0.1=11$ photo-electrons $/ \mathrm{MeV}$. The average minimum ionising particle traversing a $2 \mathrm{~cm}$ bar perpendicularly would therefore produce a signal of $\approx 40$ photo-electrons, corner-clipping particles giving proportionately less. This value of $Y_{l}$ is sufficient to ensure that any inefficiency due to statistical fluctuations of the number of photo-electrons is negligible. More careful estimates were made using a Monte Carlo (MC) programme which tracked blue photons from the point of production by a muon to eventual photo-electron production and correctly included all geometrical and loss factors. The results of the MC programme indicated essentially the same light yield. In particular, the result of $\epsilon_{W} \sim 0.37$ confirmed the above estimate. Other processes such as imperfect optical coupling and light loss due to the bending of the WLS fibres would reduce the light yield. The latter process was the subject of a separate study [11] and indicated that a radius of curvature to fibre radius ratio of greater than 65 results in a light loss of less than $10 \%$ with the loss occurring in a transition region at bending angles $\Phi \sim \pi / 8 \mathrm{rad}$. 


\subsection{Discriminators}

The requirement of minimum power consumption excluded the use of fast digitisation of the PMT signals; instead the signals would be digitised by simple discriminators. It would be advantageous to use a common threshold for all channels. Once allowance is made for the gain difference between the PMT pixels and the requirement of efficiency for minimum ionising particles, the discriminator thresholds must be equivalent to a few photo-electrons, but greater than one photo-electron to eliminate PMT dark noise and single photons from cross-talk. Since the signals from the PMTs were expected to be small and the power consumption of fast pre-amplifiers was unacceptable, the design required the analogue signals from the PMTs to be detected by lowthreshold discriminators without amplification. With a PMT gain of $10^{6}$ a single photo-electron would develop a $3.5 \mathrm{mV}$ signal if integrated directly on $47 \mathrm{pF}$. Commercial comparators can operate with threshold voltages as low as $5-10 \mathrm{mV}$ if proper precautions are taken to eliminate extraneous noise.

A further consideration for the design of the electronics was that the full photoelectron signal equivalent to the estimated light yield is not available unless the signal is fully integrated before discrimination. The time distribution of the photo-electrons is determined by the decay time of the flours in the WLS fibre $(7-10 \mathrm{nS})$ and the collection time of the blue photons, estimated to be another $5-10 \mathrm{nS}$ in this geometry. An integration time of $\approx 100 \mathrm{nS}$ or greater is required to achieve the full signal. This requirement conflicts with the detector requirement that the discriminators are able to re-trigger after $200 \mathrm{nS}$ for a wide range of signals. With these considerations in mind, lowthreshold discriminators, mounted directly on the base of the photomultipliers, were developed.

\section{Light Yield and Light Attenuation Measurements}

\subsection{Light Yield Measurements}

The study of light yield was performed using single- and double-clad $1.2 \mathrm{~mm}$ diameter Bicron BCF-91A WLS fibres embedded in bars of Bicron BC-400 [12] polyvinyltoluene-based plastic scintillator (refractive index $n=1.58$ ). The $\mathrm{BCF}$ fibres have a polystyrene core of refractive index $n_{\text {core }}=1.6$. The singleclad fibres (BCF-91A SC) have a thin polymethylmethacrylate (PMMA) cladding of refractive index $n_{\text {clad }}=1.49$, the double-clad fibres (BCF-91A DC) have an inner PMMA cladding and an outer fluorinated polymethacrylate

cladding of refractive index $n_{\text {clad }}^{\prime}=1.42$. Double-clad fibres offer a significant 
improvement over single-clad fibres since a larger fraction of light is trapped in the fibre core. The $280 \mathrm{~mm}$ long scintillation bars had a cross section of $20 \times 35 \mathrm{~mm}^{2}$. The fibres were located inside $1.5-2.0 \mathrm{~mm}$ deep and $1.5 \mathrm{~mm}$ wide grooves which had been filled with the optical clear epoxy Bicron BC600 . The groove depth exceeded the fibre diameter by $0.5-0.8 \mathrm{~mm}$. Once the glue was set, the fibre ends were polished and the scintillator bars painted with Bicron BC-620, a diffuse white reflector composed mainly of titanium dioxide, $\mathrm{TiO}_{2}$.

The WLS fibres were taken to an M16 and positioned by guidance holes in a block of Tufnol high pressure laminate fitting the outer dimensions of the PMT. Each fibre was centred on the centre of a single photocathode pixel. The fibres were glued into the holes using a fast epoxy, and coupled optically to the photocathode using optical grease. The anode signals from the M16 were amplified using an amplifier with a $10 \mathrm{nS}$ integration time constant and a gain of $\sim 6$. The output signals from the amplifier were discriminated and counted. The M16 was operated with an HV of -850 Volts. The counting rate was measured as a function of threshold voltage when the bars were illuminated with a weak $(2 \mathrm{k} \mathrm{Bq}){ }^{106} \mathrm{Ru} \beta$ source. The maximum energy of the electrons emitted by ${ }^{106} \mathrm{Ru}$ is $3.54 \mathrm{MeV}(78 \%)$ with sub-dominant contributions of transitions with $2.4 \mathrm{MeV}(11 \%)$ and $3.0 \mathrm{MeV}(8 \%)$ maximum energy and additional gamma lines.

The anode counting rate, which varied between $1000 \mathrm{~Hz}$ and $100 \mathrm{~Hz}$, was measured for two different channels as a function of discriminator threshold from 30 to $300 \mathrm{mV}$. The total count rate at minimum threshold was normalised to accommodate gain variations between pixels. The peak of the single photoelectron signal was determined by measuring the counting rate-threshold curve with no source but with a controlled light leak, thereby enabling the threshold voltage to be converted to equivalent photo-electrons.

The measured counting rate-threshold curves were compared with the predictions of a MC simulation based on GEANT 3.21 [13]. In the simulation the energy depositions and particle tracks were recorded in the active and inactive materials. The assumption was made that the photo-electron output was proportional to the energy deposited in the scintillator, i.e. $N_{\mathrm{pe}}=Y_{l} E_{\mathrm{dep}}$ where $Y_{l}$ was the conversion factor ('light yield') to be determined. The experimental photo-electron resolution of the photomultiplier was simulated by a convolution of the Poisson distribution, $P\left(n_{p e} \mid N_{p e}\right)$, for the number of photoelectrons released at the photocathode with a Gaussian distribution with a width of $\sigma_{E}=\sigma \sqrt{n_{p . e .}}$ where $\sigma \approx 40 \%$ was deduced from single photoelectron measurements with the M16. A match of the slopes of the simulated and measured counting rate curves was achieved with a light yield, $Y_{l}$, of 8 photo-electrons/MeV of deposited energy as shown in Fig. 3. Allowing for the attenuation in the WLS fibre $(T \sim 55 \%)$, the measured light yield was 
consistent with the estimate of 11 photo-electrons/MeV given in Section 3.

Measurements also showed that a white diffuse reflector (BC-620 paint) at the open end of a fibre allowed the collection of secondary photons propagating away from the PMT and led to an increase in light yield of $\sim 20 \%$. A further increase was sought by using an aluminised Mylar (a polyester film) foil, which would give specular reflection, but no significant change was observed. A possible explanation could be a misalignment of the reflector foil with respect to the fibre axis. These results are shown in Table 1.

We concluded that the light yield of a such a scintillator - WLS fibre combination is adequate for an efficient detector and that it will allow the use of low power electronics.

\subsection{Light Attenuation Measurements}

Light attenuation in the WLS fibres was measured by illuminating individual, loose fibres with a light-emitting diode. The LED, which had a peak wavelength of $420 \mathrm{~nm}$, was placed so as to shine perpendicularly onto a WLS fibre at a variable distance from the end coupled to a PMT. The LED was fixed to a housing which could move smoothly over the tested fibres. Initially, the far end of the WLS fibres were covered with a black absorber to remove all secondary light. The green photons were detected by a 2 inch Philips XP-2230 photomultiplier tube. The average PMT anode current was determined by measuring the voltage drop across $10 \mathrm{M} \Omega$ in parallel with $12 \mu \mathrm{F}$. The intensity of illumination of the fibre was controlled by varying the current through the LED. The PMT output varied linearly with LED current over most of the working range, with non-linearities at the low and high ends due to the non-linearity of the LED and saturation (droop) of the PMT output current respectively.

As well as measuring the PMT anode current the counting rate of discriminated PMT output pulses was also measured. An extremely good correlation between count rate and anode current was measured because the incoherent illumination of the fibre by the LED produced a succession of single photoelectrons at the PMT cathode. Both anode current and counting rate were used as a measure of the total light intensity.

It was necessary to turn on the LED one hour before any measurements to allow the system to become fully stabilised. After this time the variations in anode current for fixed illumination did not exceed $2 \%$. All the detector components were located in a box providing light tightness and mechanical protection. After exposure of the photomultipliers to ambient light, such as fluorescent room lighting when changing the experimental set-up, a tempo- 
rary increase in anode dark current was observed. The recovery time of the photomultiplier tubes was of the order of 20 - 60 minutes, depending on the exposure. After such a waiting period a series of readings were taken for each measurement. As a cross-check of any long term effects, the light box was sealed on several occasions for 16 hours before turning on the $\mathrm{HV}$ of the PMTs.

The light intensities transmitted over distances of up to $3 \mathrm{~m}$ were measured for single- and double-clad fibres. Fig. 2 shows the dependence of the light intensity ( $\mathrm{dc}$ anode current) as a function of photocathode distance from excitation source, $d$, for different fibres. The data shown in Fig. 2 demonstrates that the simple assumption of a single attenuation length is incorrect. The measurements are best described by a sum of two exponential functions: $I_{p h}=I_{L} e^{-d / \lambda_{L}}+I_{S} e^{-d / \lambda_{S}}$, where $\lambda_{L}$ represents a longer attenuation length, $\lambda_{S}$ a shorter one and $I_{L / S}$ are the relative intensities. We observed that the short attenuation length dominated the light losses for distances $d<1 \mathrm{~m}$ while the longer attenuation length was appropriate for distances of $d \approx 1-5 \mathrm{~m}$ or more. Results for best fits on the measured intensities are shown in Table 2.

Most light detected within the first $50 \mathrm{~cm}$ from the light source is referred to as cladding light, which is not trapped in the fibre core but by total internal reflections at the cladding-air interface. It is usually a factor 3-4 more intense than core light. The transmission of cladding light is strongly affected by the surface quality of the fibre: cracks in the cladding or defects in the surface can cause significant light losses leading to differences in the light yield of otherwise identical fibres. Due to the unavoidable degradation of the fibre surface, cladding light is expected to be attenuated more strongly than core light. Since cladding light is attenuated by an unpredictable amount, variations of its contributions to the total light intensity are expected. To achieve an understanding of the transmission of light in the core of WLS fibres, the removal of the cladding light is useful. This was accomplished by coating the fibre with an extra-mural absorber $(E M A)$. The second set of data in Table 2 shows the results of these measurements. Since the LED housing prohibited the covering of the first $5 \mathrm{~cm}$ of fibre, the fits to the data points have been constrained by $I_{S}=R I_{L}$, where $R$ is the ratio of light intensities at $d \simeq 5 \mathrm{~cm}$ as given by the first set of measurements. This method allowed the estimation of the relative intensities of the two light components at zero fibre length. For double clad fibres a higher $I_{L} / I_{S}$ ratio was observed which could be explained by a lower proportion of light propagating by reflections at the exposed surface. Errors on the attenuation lengths include an estimated systematic error taking into account uncertainties due to possible drifts of the output signals and the effects of irregularities in the fibres. The results on the long attenuation length were less accurate because the EMA was only applied to a length of $d \approx 1 \mathrm{~m}$ of fibre. Within the accuracy of the measurements it can be concluded that the short attenuation length decreased by around $30 \%$, whereas the long 
attenuation length was not significantly affected by the addition of the EMA.

The improvement in trapping efficiency and attenuation length of double-clad fibre with respect to single-clad fibre can be seen in Fig. 2. For fixed illumination by the LED at $d=3 \mathrm{~m}$ (where the cladding light has been substantially attenuated) the PMT output currents are in the ratio of $\sim 3.8: 2.0$ (=1.9) for the double- and single-clad fibres respectively.

\section{Prototype Hodoscope}

\subsection{Hodoscope Construction}

A prototype two-plane hodoscope was built to verify the design. The planes and scintillator bars had the same dimensions as those foreseen for the full detector, but a somewhat different mechanical arrangement for ease of access and testing. The two planes were orthogonal and each contained eight scintillator bars of $20 \times 35 \mathrm{~mm}^{2}$ cross-section and $280 \mathrm{~mm}$ long. The whole was enclosed in a single rigid aluminium box approximately $600 \mathrm{~mm}$ square.

The scintillator strips were mounted on either side of a $3 \mathrm{~mm}$ thick central aluminium sheet in a box consisting of a 'picture frame' made of $50 \times 8 \mathrm{~mm}$ aluminium bars. The central sheet slotted into grooves machined in the bars. This method of construction provided considerable rigidity for relatively little weight. The top and bottom of the box were closed with thin aluminium covers. An M16 PMT and discriminator cards, mounted directly on the PMT base, were located in a demountable external housing.

A $1.2 \mathrm{~mm}$ diameter WLS fibre was embedded to a depth of $\sim 1.8 \mathrm{~mm}$ in each scintillator bar. Double-clad fibres (Bicron BCF-91A) were used for the top hodoscope and single-clad fibres for the bottom hodoscope. On leaving the scintillator bars the fibres were loosely enclosed in opaque sleeving to prevent optical cross-talk and brought to a Tufnol 'cookie' on the side of the hodoscope box. Holes in the cookie aligned with the pixels of the PMT.

A photograph of the aluminium frame with the top hodoscope plane and the fibre routing is shown in Fig. 4. The bottom hodoscope plane is hidden below the central sheet. 


\subsection{Electronics}

Signal discrimination was provided by low threshold, high speed circuits. Two boards carrying eight channels of discriminators were mounted directly onto the PMT base which also carried the divider chain for the PMT dynodes. The circuit diagram of one channel is shown in Fig. 6. The discriminators used Maxim 903 comparators. These have separate power supply connections for the analogue input and digital output stages which is an advantage if the lowest possible thresholds are to be achieved. Simple $R C$ integration of the PMT anode signal is provided at the discriminator input, the time constant of $10 \mathrm{nS}$ being chosen to match the estimated light-collection time of scintillator - WLS fibre combination. The relatively large value of $47 \mathrm{pF}$ was chosen for the input capacitance in order to reduce capacitative cross-talk. Independent externally adjustable thresholds were provided in common for even and odd channels. The $220 \mathrm{k} \Omega$ feedback resistor provided $5 \mathrm{mV}$ of hysteresis. The lowest practical threshold which could be achieved was $\sim 6 \mathrm{mV}$ at the discriminator input.

Since the PMT signal due to many photo-electrons from the scintillators arrives over a time of $\sim 10 \mathrm{nS}$, the $R_{i n} C_{\text {in }}$ integration time constant of $10 \mathrm{nS}$ at the input of the discriminators provides only partial integration. The input signal to the discriminators will depend non-linearly on the number of photoelectrons. An isolated photo-electron will produce an input signal of $V_{\text {in }}\left(1_{\mathrm{pe}}\right)=$ $e G / C_{i n}$ (where $e$ is the electron charge and $G$ is the PMT gain) whereas the input voltage from many $\left(N_{p e}\right)$ photo-electrons will tend to $V_{i n}\left(N_{\text {pe }}\right)=$ $N_{\text {pe }} e G \exp (-1) / C_{i n}$.

The TTL output stages of the discriminators were connected to drivers which transmitted the Low Voltage Differential Signals (LVDS) to an external interface board via 34-way 'flat-'n-twist' cables. The same cables were also used to provide the analogue and digital power, test signals and the common thresholds to the discriminators.

A state-machine programmed ' $\mathrm{MACH}$ 435' PGA chip managed the trigger logic on the interface board and also performed handshaking with a PC via a general purpose PCI card. The logic was designed to latch the signals from the M16 on receipt of a trigger. Triggers could be derived from external scintillation counters or internally by OR-ing signals from the M16. Since the trigger signal from the external conventional scintillation counters was late with respect to signals from the M16 (due to the transit times in the PMTs), the discriminator signals were stretched by up to $200 \mathrm{nS}$, in $25 \mathrm{nS}$ steps in the PGA.

A LabView Virtual Instrument (VI) application running on the PC was used 
for on-line analysis of the data and to write the data to files for further analysis. The data acquisition scheme for the hodoscope is shown in Fig. 7. The data samples read by the PC consisted of bit patterns of 16 channels, each channel representing a corresponding scintillator. These bit patterns were used for the two-dimensional track reconstruction and for the determination of the acceptance and efficiency of the hodoscope.

The hodoscope was mounted into a cosmic ray test stand. Two (three) scintillator paddles of size $584 \times 216 \mathrm{~mm}^{2}$, directly coupled to Philips XP-2230 photomultipliers, were located above and below the hodoscope. In addition, two lead shields, a larger block of $5 \mathrm{~cm}$ height and $41 \mathrm{~cm}$ length and a smaller one of $2.5 \mathrm{~cm}$ height and $63 \mathrm{~cm}$ length, were stacked between the scintillator paddles. A schematic drawing is shown in Fig. 5. Minimum ionising muons lost a minimum of $\Delta E \approx 100 \mathrm{MeV}$ kinetic energy in the absorbers. A cosmic ray trigger was derived from the overlap coincidence of the signals.

\subsection{Geometric Factor and Intrinsic Efficiency}

The cosmic ray trigger had an area a few times greater than the active area of the hodoscope and accepted particles over a wide range of angles. The geometrical efficiency of the top (bottom) hodoscope plane was approximately 25 (12)\% for the two scintillator trigger and 20 (14)\% for the three scintillator trigger. It was obvious from the orientation of the hodoscope strips (see Fig. 5) that the top plane scintillators accepted particles over a wider range of angles. A MC simulation of the low energy cosmic ray flux was used to determine the intrinsic efficiency of the scintillator strips and light yield for minimum ionising muons.

The acceptance, $\alpha$, of the hodoscope for detecting an incident muon was factored into two parts, the intrinsic efficiency, $\epsilon$, and the geometrical factor, $(d A d \Omega)$. The acceptance is simply the product of the two. The intrinsic efficiency is the ratio of particles detected by the hodoscope to the number of particles incident on area $d A$ from solid angle element $d \Omega$. The geometrical factor was deduced from the simulation. The differential and integral spectra of cosmic rays were taken from [14] and [15]. A minimum momentum cut of $0.2 \mathrm{GeV} / c$ was used to allow for the two lead shields. In the simulation, muons were generated on a virtual sphere enclosing the hodoscope-trigger counter assembly. The points on the virtual sphere were distributed so that any particle within an element of solid angle, $d \Omega$, traversed in a downward sense a horizontal element of area $d A$. The zenith angle dependence of the flux was taken to be $I(\theta)=I\left(0^{\circ}\right) \cos ^{2}(\theta)$. Azimuthal symmetry was assumed.

The HV applied to the photomultiplier and the threshold on the discriminator 
cards were chosen to achieve a high intrinsic efficiency and low cross-talk. The plateau curves for thresholds of $45-55 \mathrm{mV}$ showed a minimum slope at around $-850 \mathrm{~V}$, where the counting rate was the least sensitive to drifts in the HV.

The geometric factor could be eliminated by requiring a coincident hit in one hodoscope planes to analyse the other. These global efficiencies for each hodoscope plane, defined as $\epsilon_{T(B)}=\Sigma T(B) /\left(T_{\mathrm{ext}} \cdot \Sigma B(T)\right)$, are shown in Fig. 8 as a function of HV and threshold voltage. The plateau voltage for the top plane, which used double-clad WLS fibres, was $~ 50 \mathrm{~V}$ lower than for the bottom plane, consistent with the greater light-yield expected for double-clad fibres. The efficiency of each plane was determined at a high voltage of $-850 \mathrm{~V}$, corresponding to a PMT gain of $3 \times 10^{6}$, and varying threshold voltages. The measured acceptances and global efficiencies are given in Table 3 . The average efficiency was $\epsilon \approx 98.6 \%$ for a threshold of $45 \mathrm{mV}$, corresponding to $10-12$ photo-electrons.

Due to the geometry of the scintillator paddles the illumination of the hodoscope by cosmic ray muons was non-uniform. Fig. 9 shows the relative acceptance, that is the fraction of hits per trigger in each scintillator bar, for two sets of measurements and the corresponding simulation values. The small asymmetry in direction of the paddles' axes is due to their asymmetric set-up. In the perpendicular direction the limited accuracy in the positioning of the paddles led to a small left-right asymmetry. To check the efficiency of the two outermost scintillator bars, which exhibited low count rates because of their small geometric acceptance, the hodoscope was moved $5 \mathrm{~cm}$ in either direction. The relative acceptance of the two counters is shown in Fig. 9 by squares.

The global efficiencies, as defined above, include muons which clip the corners of the hodoscope strips. To deduce the true intrinsic efficiency for muons passing through at least $2 \mathrm{~cm}$ of scintillator in each plane, the $\mathrm{MC}$ simulation was used to generate a photo-electron spectrum for the cosmic ray flux. The simulation included the gaps between the scintillators due to their individual paint coating and their finite thickness. Fig. 10(a) shows the simulated distribution of the number of photo-electrons spectrum for the scintillator bars. There is a significant tail at low numbers of photo-electrons due to the corner clipping muons. An estimate of the minimum light yield of the hodoscope was possible from the measured efficiencies $\epsilon \approx 98 \%$. Fig. 10(b) shows a calculation of the global efficiencies, including corner-clipping muons, versus threshold, for different light yields. Since, after allowance for the difference in gain between pixels, the largest effective threshold was 10-12 photo-electrons, we conclude from Fig. 10(b) that the light yield of the hodoscope elements was larger than 8 photo-electrons $/ \mathrm{MeV}$, in good agreement with the estimates given in Section 3. This translates into an absolute light yield of $N>28$ photo-electrons for a normally incident minimum ionising muon with an average energy deposition of $3.5 \mathrm{MeV}$. 


\subsection{Multiplicity}

At an operating $\mathrm{HV}$ of $-850 \mathrm{~V}$ and a threshold of $45 \mathrm{mV}$, the hit multiplicities were measured to be $\langle N\rangle_{T O P}=1.13$ and $\langle N\rangle_{B O T}=1.10$ for the three scintillator trigger, compared with $\langle N\rangle_{T O P}=1.01$ and $\langle N\rangle_{B O T}=1.03$ expected from the simulation. The $\sim 10 \%$ excess multiplicity was attributed to crosstalk in the PMT and some electronics cross-talk. Cross-talk in the PMT can be optical, which occurs due to the divergence of light as it passes through the borosilicate glass window, or electronic, which occurs if electrons begin the multiplication process in the wrong dynode chain. We attributed the proportion of hits in non-neighbouring channels $(\sim 1 \%)$ to random coincidences with background radiation.

\section{Summary and Discussion}

A scintillator hodoscope has been developed to demonstrate a possible detector concept for the measurement of the low energy muon flux in an aircraft. The proposed detector (ADLER) had to be self-contained, as small and light as possible, consistent with the design objective and consume minimum electrical power. Our solution is based on bars of plastic scintillation counters read out via WLS fibres and multi-anode photomultiplier tubes. The multi-anode PMT Hamamatsu R5900-00-M16 requires little power, is compact in size, and has stable properties that make it suitable for our use with scintillating fibres. In order to optimise the fibre routings inside the hodoscope, the light attenuation characteristics of WLS fibres, parameterised by two different attenuation lengths, have been measured. Extra-mural absorbers applied to the fibre surface were successfully used to measure the amount of cladding and core light in the fibres. Simple, low power, low-threshold, discriminators were developed. Two boards with eight discriminators each were directly mounted on the the base of the photomultiplier tube, which also carried the divider chain for the PMT dynodes.

A prototype scintillator hodoscope was constructed and tested with cosmic rays. Data acquisition was performed without standard electronic crates and modules, but with a single PGA chip and a high speed digital I/O board of a PC. The intrinsic efficiency of the hodoscope was found to be above $98 \%$. Average hit multiplicities per plane were found to be $\sim 10 \%$ higher than expected, suggesting the presence of some optical and electronic cross-talk. The inferred light yield of $>8$ photo-electrons/MeV was entirely adequate for the intended purpose of the detector. 


\section{Acknowledgements}

We thank the MINOS group at the University of Oxford for lending us the M16 and two voltage divider boards. We are also much indebted to the technical staff, in particular to M. Dawson, for their assistance. We would like to thank K. Ruddick of the University of Minnesota for the programme to estimate light yields in scintillators.

This research was supported by the UK Particle Physics and Astronomy Research Council (PPARC).

\section{References}

[1] C. P. Achenbach and J. H. Cobb, A new airborne detector for atmospheric muons, in: K.-H. Kampert, G. Hainzelmann, C. Spiering (Eds.), Proc. of the 27th Int. Cosmic Ray Conf. (ICRC 2001), Copernicus Ges., 2001, 1313-1316.

[2] G. Barr, T. K. Gaisser, T. Stanev, Flux of atmospheric neutrinos, Phys. Rev. D39 (1989) 3532-3534.

[3] V. Agrawal, T. K. Gaisser, P. Lipari, T. Stanev, Atmospheric neutrino flux above $1 \mathrm{GeV}$, Phys. Rev. D53 (3) (1996) 1314-1323.

[4] M. Honda, K. Kasahara, K. Hidaka, S. Midorikawa, Atmospheric neutrino fluxes, Phys. Lett. B248 (1990) 193-198.

[5] Y. Fukuda (Super-Kamiokande coll.), et al., Evidence for oscillation of atmospheric neutrinos, Phys. Rev. Lett. 81 (1998) 1562-1567.

[6] M. Ambrosio (MACRO coll.), et al., Measurement of the atmospheric neutrinoinduced upgoing muon flux using MACRO, Phys. Lett. B434 (1998) 451-457.

[7] W. W. M. Allison (Soudan-2 coll.), et al., The atmospheric neutrino flavor ratio from a 3.9 fiducial kiloton year exposure of Soudan-2, Phys. Lett. B449 (1998) $137-144$.

[8] Hamamatsu Photonics UK, Photomultiplier Tubes and Assemblies For Scintillation Counting \& High Energy Physics (2000).

[9] Y. Yoshizawa, J. Takeuchi, The latest vacuum photodetector, Nucl. Instr. and Meth. in Phys. Res. A387 (1997) 33-37.

[10] M. Enkelmann, U. Werthenbach, G. Zech, T. Zeuner, An optical readout for a fiber tracker, Nucl. Instr. and Meth. in Phys. Res. A412 (1998) 216-222.

[11] C. P. Achenbach and J. H. Cobb, Computational studies of light acceptance and propagation in straight and curved multimodal active fibres, J. Opt. A: Pure Appl. Opt. 5 (2003) 239-249. 
[12] Bicron, Organic Scintillators, Related Materials and Detectors (1997).

[13] Application Software Group, Geant Detector Description and Simulation Tool, CERN Program Library Long Writeup W5013 (1994).

[14] P. K. F. Grieder, Cosmic rays at earth, Elsevier Science, 2001.

[15] O. C. Allkofer, K. Carstensen, W. D. Dau, The absolute cosmic ray muon spectrum at sea level, Phys. Lett. 36 B (1971) 425-427. 
Table 1

Relative counting rates of scintillator - WLS fibre combinations measured with a simple discriminator and scaler set-up. Diffuse (paint) and specular (aluminised Mylar) reflectors were used at the open end of the fibre. Results for two different discriminator thresholds are shown.

\begin{tabular}{ccccc}
\hline$V_{t h r}$ & open & 1st paint & 2nd paint & Mylar \\
\hline \hline $30 \mathrm{mV}$ & 1. & 1.19 & 1.14 & 1.13 \\
$40 \mathrm{mV}$ & 1. & 1.22 & 1.18 & 1.16 \\
\hline
\end{tabular}

Table 2

Parameters of double exponential fits to counting rate measurements with single and double clad WLS fibres. The second set of measurements included an extramural absorber $(E M A)$.

\begin{tabular}{lcccc}
\hline Cladding & $I_{L}$ & $\lambda_{L}(\mathrm{~cm})$ & $I_{S}$ & $\lambda_{S}(\mathrm{~cm})$ \\
\hline \hline single & $9.1 \pm 0.9$ & $203 \pm 22$ & $14.3 \pm 0.8$ & $32 \pm 2$ \\
double & $10.8 \pm 0.8$ & $289 \pm 24$ & $11.3 \pm 0.6$ & $35 \pm 4$ \\
single+EMA & $7.4 \pm 0.2$ & $365 \pm 103$ & $1.51 \times I_{L}$ & $19.1 \pm 2.8$ \\
double+EMA & $9.4 \pm 0.1$ & $252 \pm 16$ & $1.13 \times I_{L}$ & $21.2 \pm 1.6$ \\
\hline
\end{tabular}

Table 3

Measured acceptances, $\alpha$, and global efficiencies, $\epsilon$, for cosmic ray muons of the two planes of the scintillator hodoscope.

\begin{tabular}{ccc}
\hline & top & bottom \\
\hline \hline$\alpha(\%)$ & 96.2 & 96.9 \\
$\epsilon(\%)$ & 98.6 & 98.7 \\
\hline
\end{tabular}




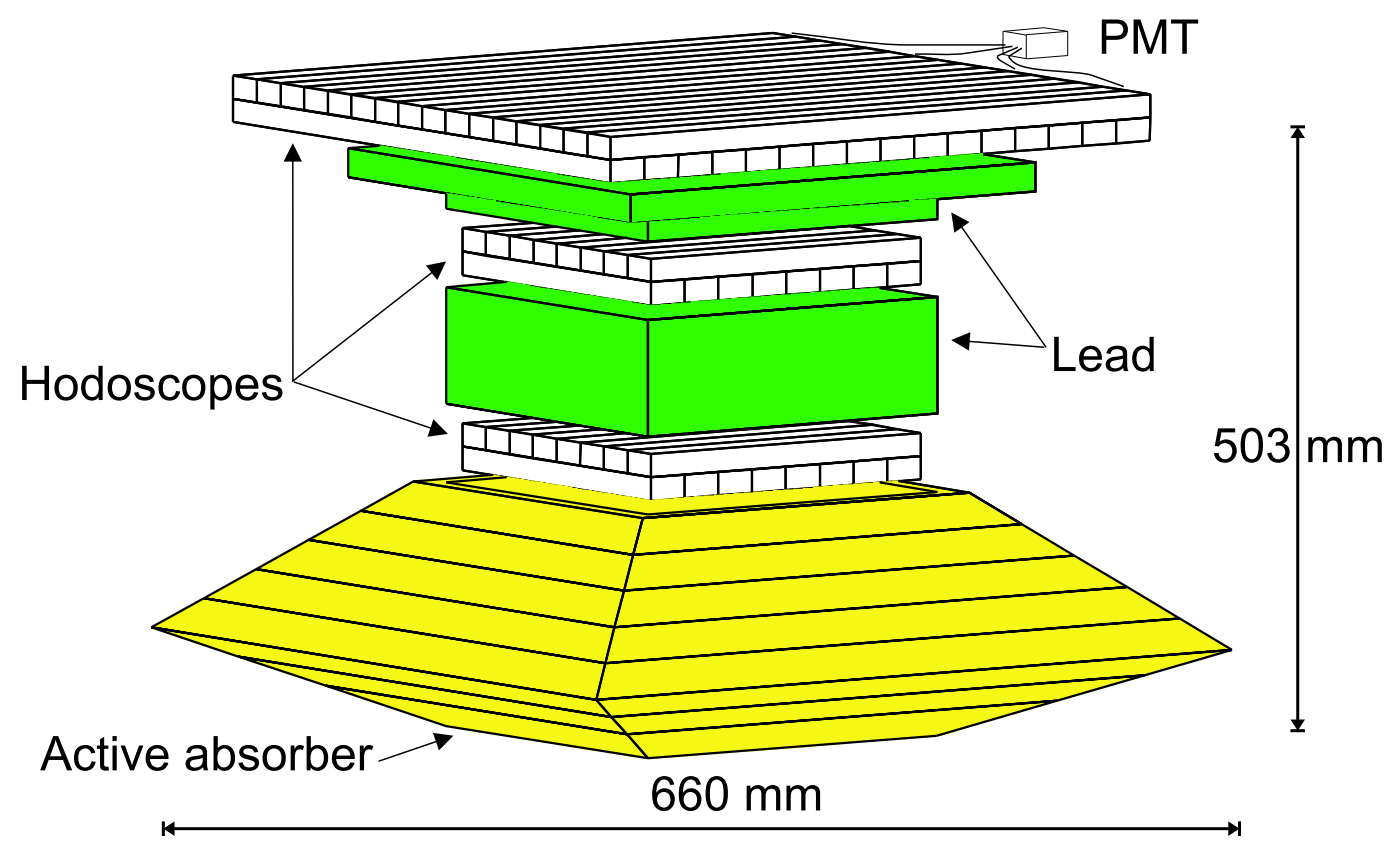

Fig. 1. Sketch of the inner components of the proposed ADLER detector which are relevant for the design of the hodoscope. Approximately 100 individual read-out channels from three hodoscopes will be needed for muon identification and track reconstruction. Six multi-anode PMTs with simple discriminator boards attached to their bases are sufficient to perform the experiment.

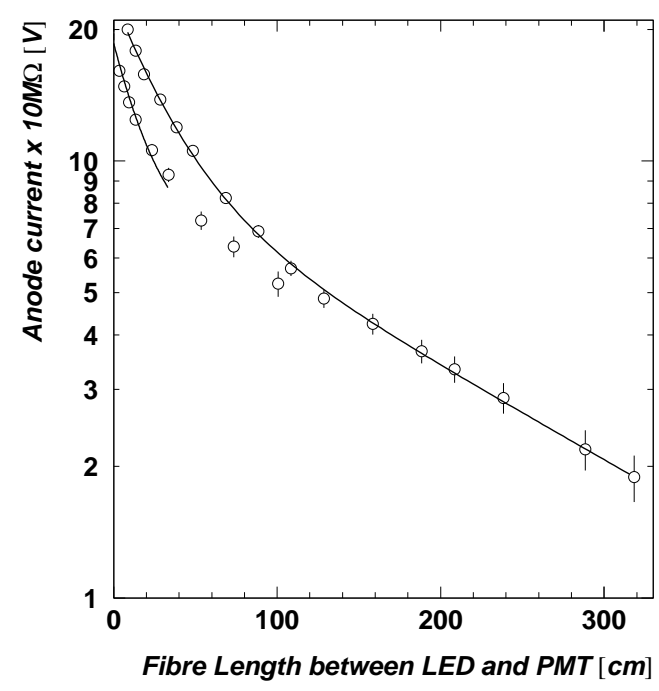

(a)

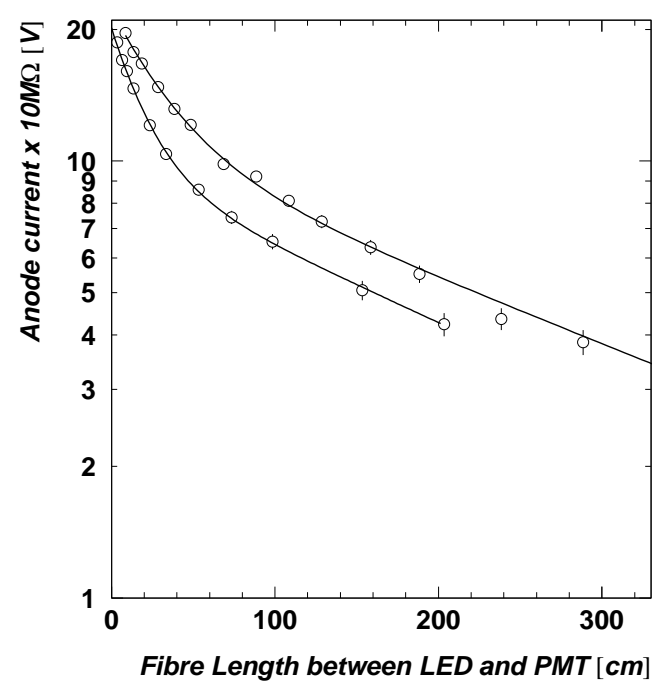

(b)

Fig. 2. Counting rate as a function of WLS fibre length. The plot shows the data points for single (a) and double clad (b) BCF-91A fibres. Short and long attenuation lengths were determined by double exponential fits. The two lower data sets were measured with fibres whose surface were covered by extra-mural absorbers (EMAs). 


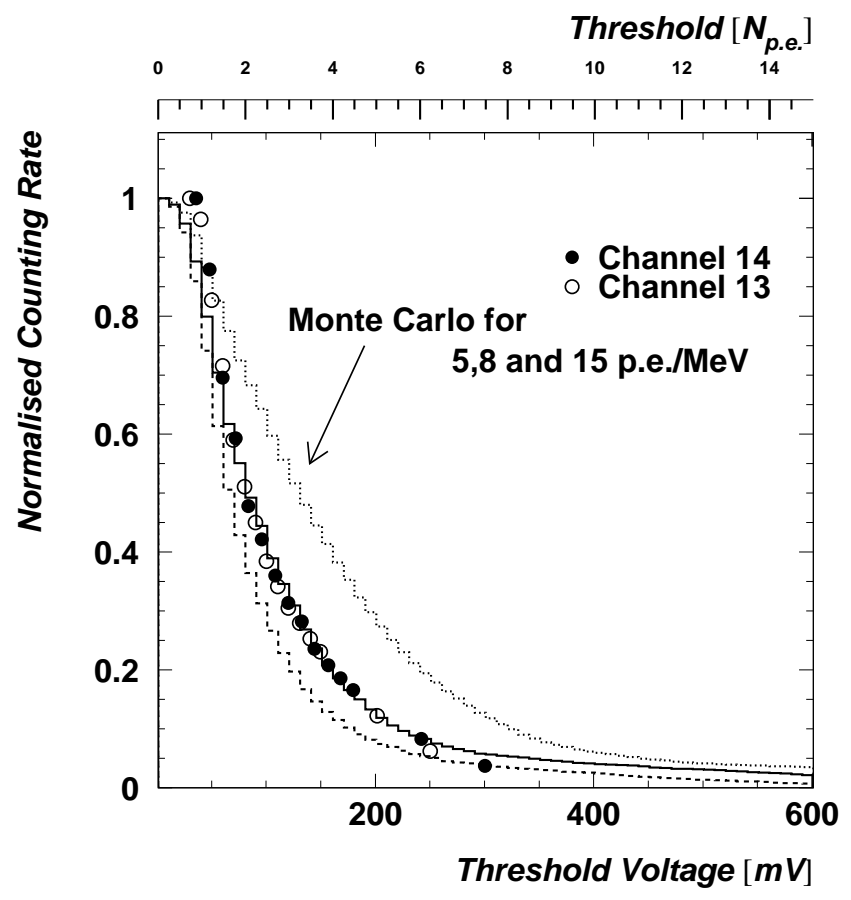

Fig. 3. Normalised counting rate as a function of discriminator threshold. The points show two measured PMT channels and the histograms show simulations for different effective light yields. The best match between measurement and simulation is achieved for a light yield of 8 photo-electrons/MeV.

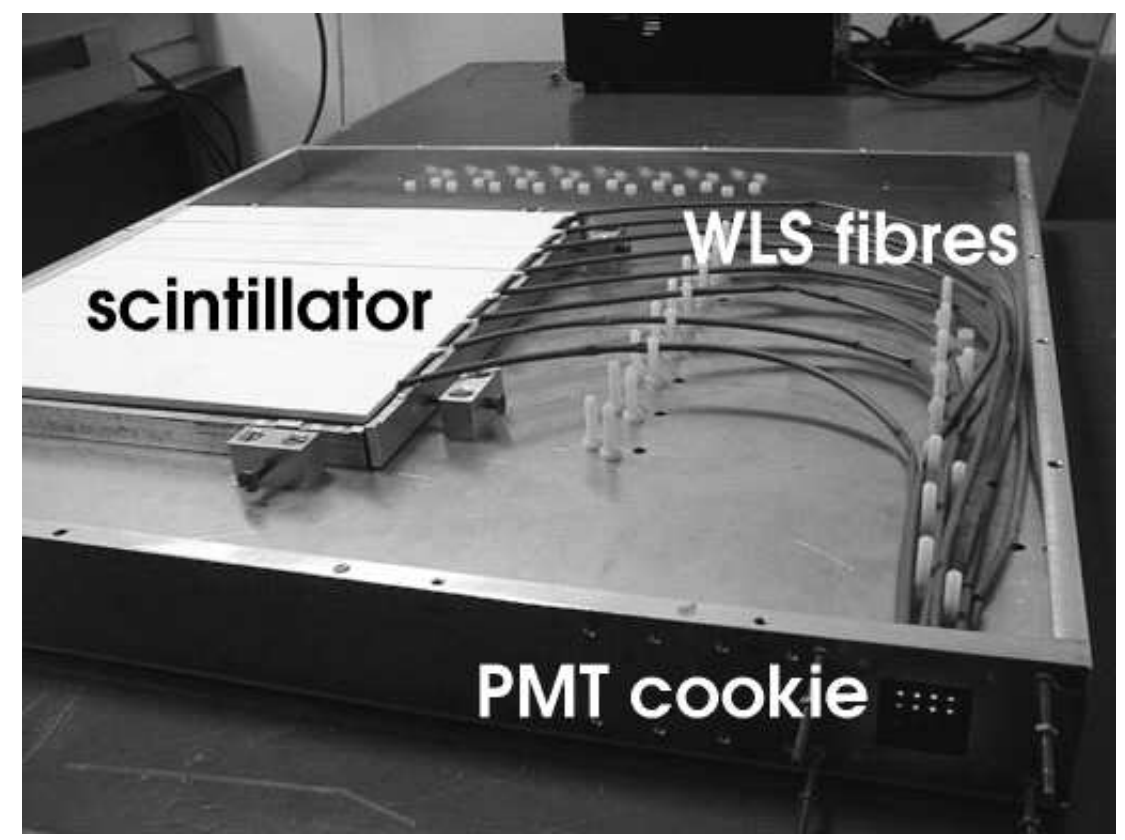

Fig. 4. Internal view of the hodoscope showing the top plane scintillators and the routing of the WLS fibres to a cookie where a PMT and two discriminator boards can be attached. The aluminium frame provided considerable rigidity for relatively little weight. 


\section{Scintillator paddle}

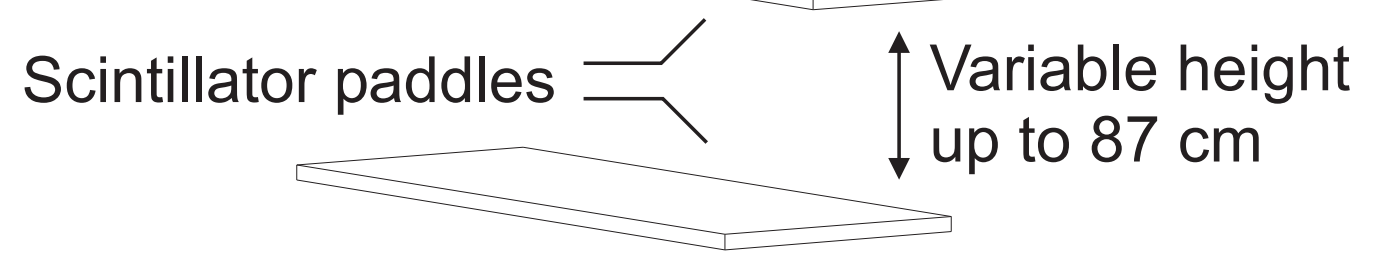

Fig. 5. Sketch of the cosmic ray test stand for the hodoscope. Three scintillator paddles and two lead shields were used to derive a clean muon trigger.

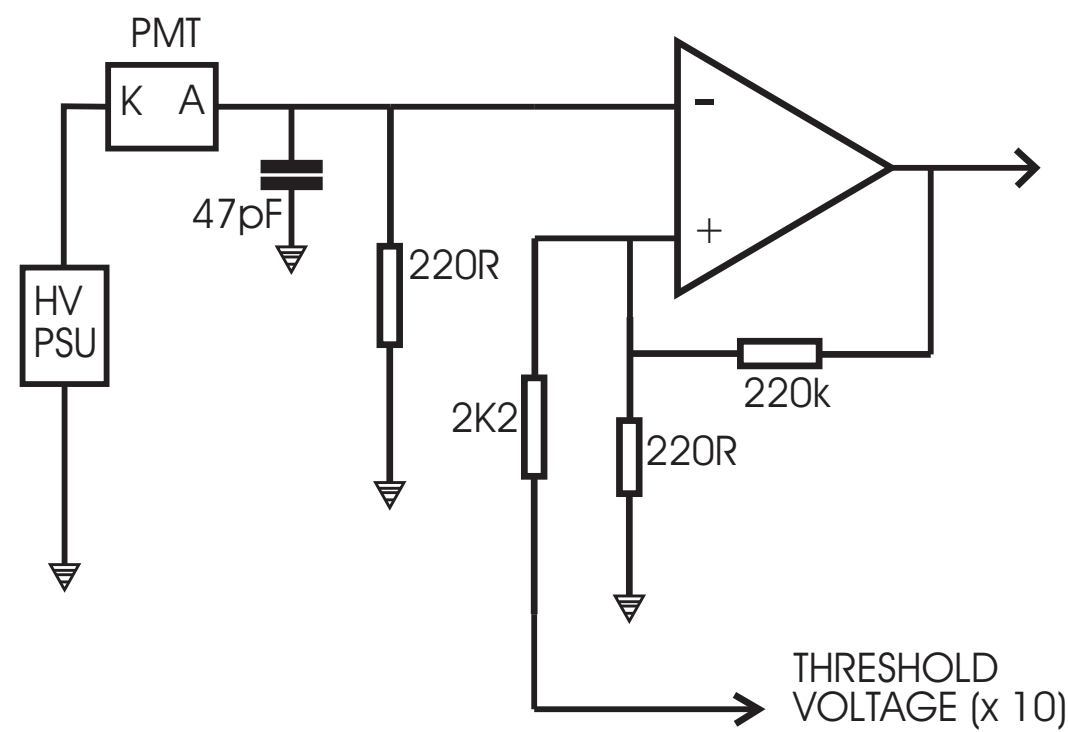

Fig. 6. One channel of the discriminator circuit. Two boards carrying eight channels were mounted directly on the base of the PMT. A common threshold voltage was provided for each group of eight channels. 


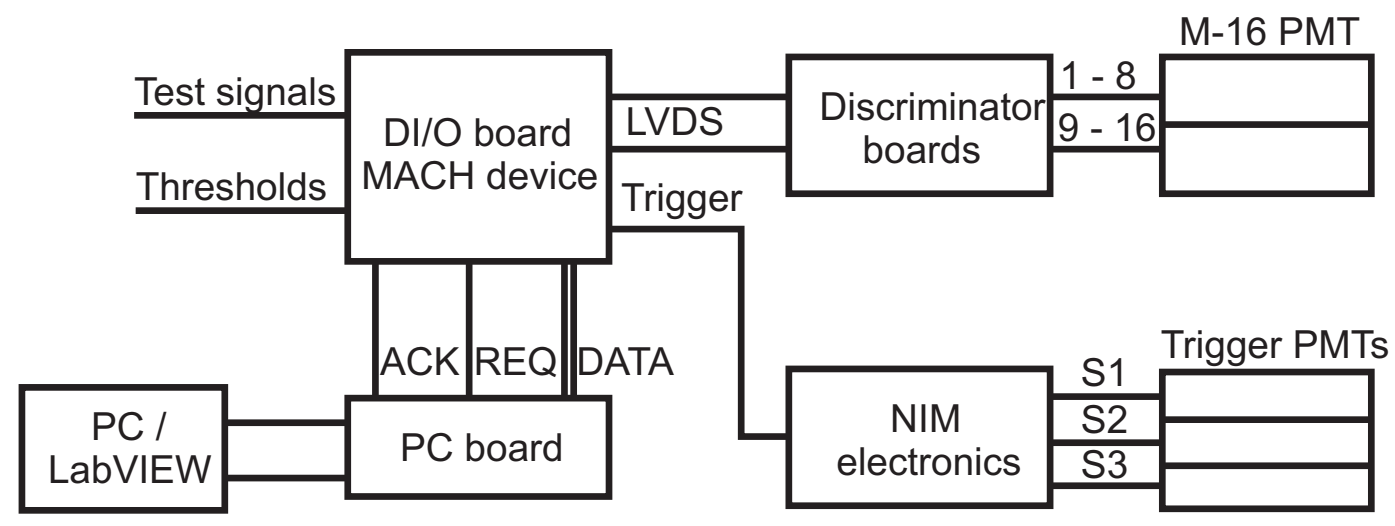

Fig. 7. The data acquisition scheme for the read-out of the hodoscope. The signals were latched from the M16 to a PC by a state machined programmed 'MACH 435' chip. Two 34-way flat-n-twist cables carry discriminator output (LVDS), the analogue and digital power, test signals and the common thresholds. The REQ and ACK lines control the handshaking. The signals of the trigger PMTs are discriminated in a NIM unit.

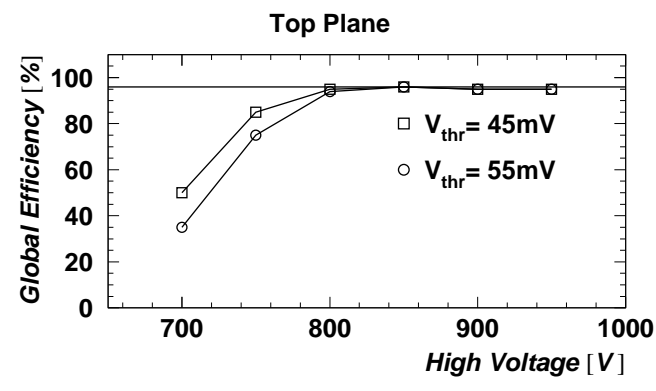

(a)

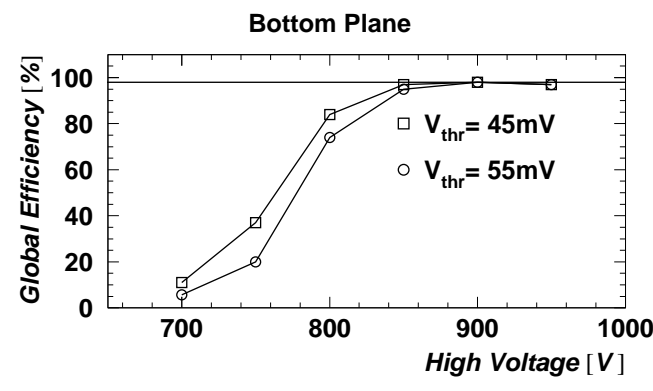

(b)

Fig. 8. Global efficiency of the top (a) and bottom (b) hodoscope plane for different HVs and discriminator thresholds. The measurements were performed with two scintillator paddles in the trigger. 

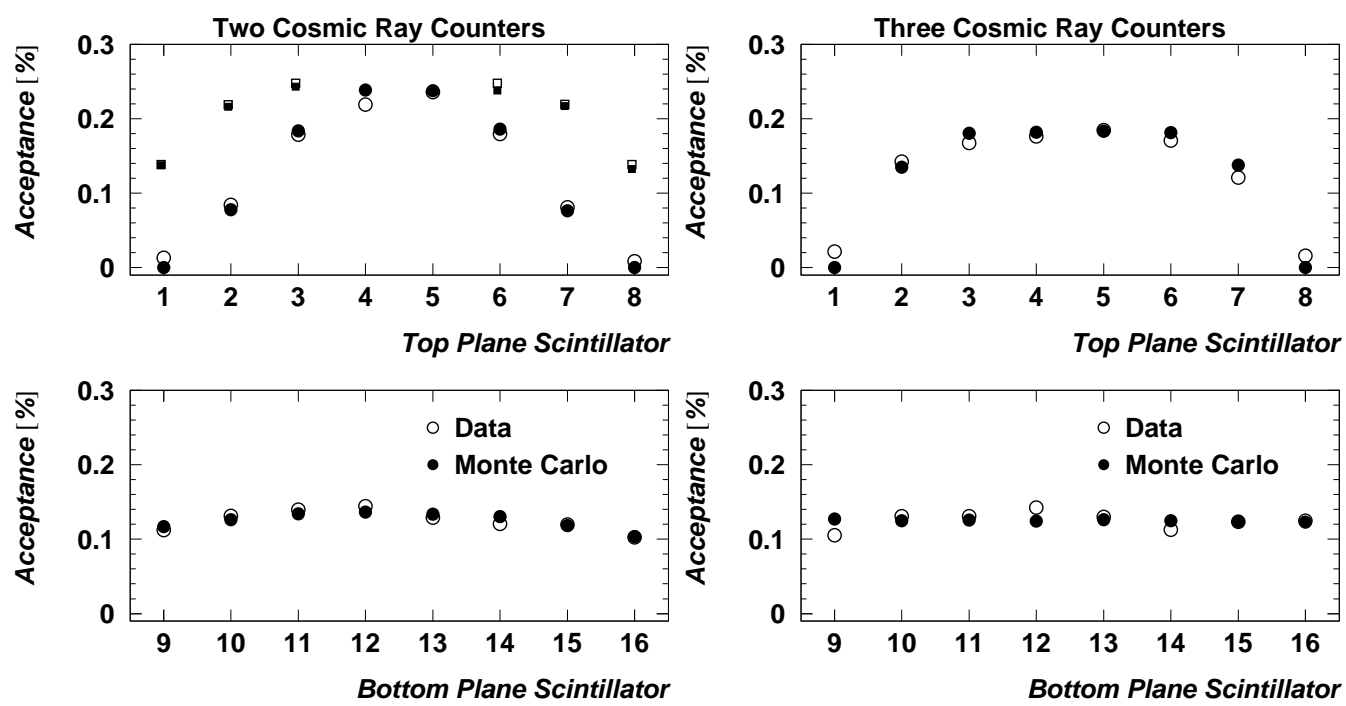

(a)

(b)

Fig. 9. Relative acceptance of the individual channels of the hodoscope for two scintillator paddles (a) and three scintillator paddles (b) in the trigger. Open circles represent measurements, full circles represent simulation. Squares show the acceptances for the hodoscope moved by $5 \mathrm{~cm}$ in either direction.

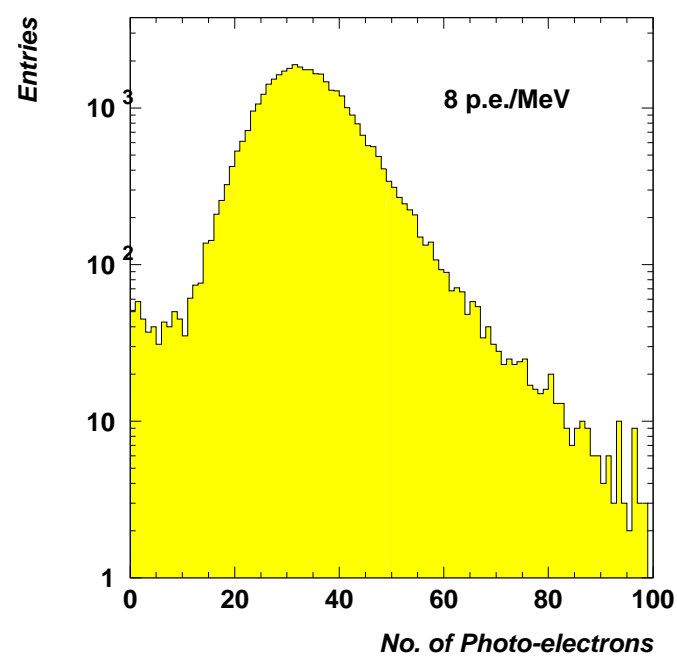

(a)

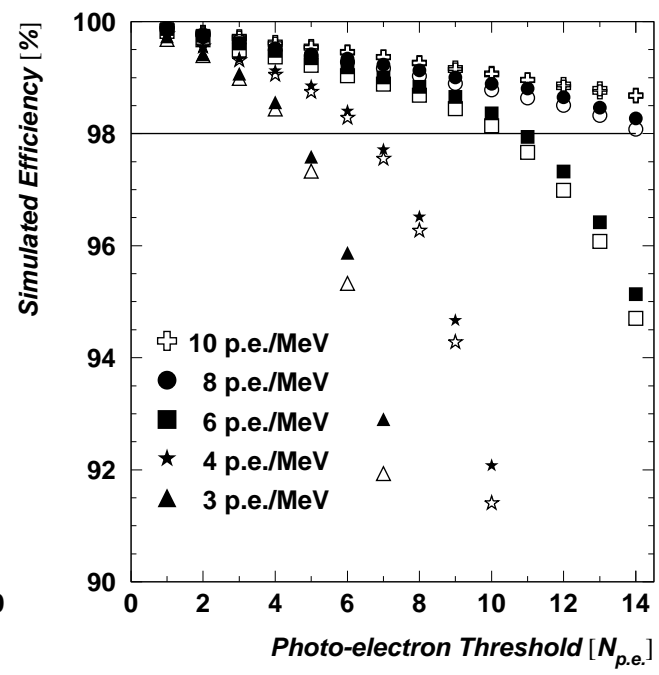

(b)

Fig. 10. Simulated photo-electron spectrum (a) for the scintillator bars and the corresponding intrinsic efficiency for detecting cosmic ray muons (b). Full and open symbols represent top and bottom plane efficiencies, respectively. The light yield of the hodoscope was determined from the measured efficiency of about $98 \%$ (horizontal line). 